Proceedings of the 16th Czech and Slovak Conference on Magnetism, Košice, Slovakia, June 13-17, 2016

\title{
Mössbauer Spectrometry Study of Biological Tissues
}

\author{
I. Bonková ${ }^{a, *}$, M. Miglierini ${ }^{b}$, M. Bujdoś ${ }^{a}$ And M. KopÁni ${ }^{c}$ \\ ${ }^{a}$ Comenius University in Bratislava, Faculty of Natural Sciences, Institute of Laboratory Research on Geomaterials, \\ Ilkovičova 6, 84215 Bratislava, Slovakia \\ ${ }^{b}$ Slovak University of Technology in Bratislava, Faculty of Electrical Engineering and Information Technology, \\ Institute of Nuclear and Physical Engineering, Ilkovičova 3, 81219 Bratislava, Slovakia \\ ${ }^{c}$ Comenius University in Bratislava, Faculty of Medicine, Department of Medical Physics, Biophysics, Informatics \\ and Telemedicine, Sasinkova 4, 81108 Bratislava, Slovakia
}

\begin{abstract}
Magnetic microstructure of iron contained in selected biological tissues is characterized and mutually compared. We have studied three types of biological samples prepared from human brain, human and horse spleen. Original samples were lyophilized (dried in a vacuum) thus providing powder forms. As a principal method of study, ${ }^{57} \mathrm{Fe}$ Mössbauer spectrometry in transmission mode was used. The Mössbauer spectrometry experiments were performed at room $(\approx 300 \mathrm{~K})$ and at liquid helium $(4.2 \mathrm{~K})$ temperature. At room temperature Mössbauer spectra show doublet-like features. Such behaviour indicates possible presence of nanoparticles with fluctuating magnetic moments that acquire arbitrary positions. On the other hand, low temperature Mössbauer spectrometry measurements demonstrate significant contribution of sextets that confirmed occurrence of blocked magnetic moments of iron-containing particles. Different relative contributions of magnetic components in the low temperature spectra for the three inspected biological tissues suggest differences in the blocking temperatures of the magnetic nanoparticles present in them.
\end{abstract}

DOI: 10.12693/APhysPolA.131.1081

PACS/topics: 33.45.+x, 87.64.kx, 31.30.Gs, 87.15.B-, 75.75.-c

\section{Introduction}

Iron is essential for life since it is required for active sites of many metalloproteins that play a key role in crucial biological processes such as oxygen transport, storage and use of oxygen in many oxidation-reduction reactions, as well as in electron transfer reactions within the cell [1].

Despite the fact that the iron in the body is indispensable, its concentration in biological tissues must be strictly controlled, because iron deficiency leads to cognitive and behavioural changes in humans and on the other hand, an increase of iron, and especially of ferrous iron, may be deleterious for living tissues as it may trigger production of free radicals [2].

Since the early days of the Mössbauer spectroscopy, studies of biological and medical materials were of close interest [2]. The first of these studies by Gonser in 1965 [3] included the Mössbauer spectra of different types of haemoglobin and continued with investigations of iron in organs as liver [4], spleen $[5,6]$ or brain $[7,8]$ by other authors. In this work, we compare magnetic behaviour of iron in selected biological tissues by the Mössbauer spectrometry.

\section{Experimental details}

The samples from a human brain, human and horse spleen were extracted post mortem at the Department of Pathological Anatomy, Comenius University in

*corresponding author; e-mail: ivana.bonkova@gmail.com
Bratislava, in accordance with the Helsinki Declaration. The samples of human brain were extracted from the region Globus Pallidus which is a part of Basal Ganglia. Fresh, soft tissues were dried in a vacuum (lyophilized) and the resulting samples were obtained in a form of powder.

Samples were analysed by ${ }^{57} \mathrm{Fe}$ Mössbauer spectrometry (MS), which was carried out using a standard constant acceleration spectrometer with a ${ }^{57} \mathrm{Co}$ source embedded in a rhodium matrix. All experiments were performed in transmission geometry at room temperature and at the temperature of $4.2 \mathrm{~K}$ using liquid helium bath cryostat. Hyperfine parameters of individual spectral components were refined by the CONFIT curve-fitting program [9]. Velocity calibration was performed by a $12.5 \mu \mathrm{m}$ thick $\alpha$-Fe foil. Isomer shifts are given with respect to a Mössbauer spectrum of the calibration $\alpha$-Fe foil taken at room temperature.

\section{Results and discussion}

The Mössbauer spectra obtained at room temperature from all investigated samples exhibit doublet-like features. They are shown in Fig. 1. At room temperature, all samples were measured in a narrow velocity range $\pm 4 \mathrm{~mm} / \mathrm{s}$ to allow better resolution of the spectral lines. Doublet-like spectra are characteristic for a nonmagnetic material that is paramagnetic at room temperature and/or are caused by superparamagnetic behaviour of small Fe-containing particles. Presence of the latter in spleen tissues was confirmed by transmission electron microscopy [10]. Particles with similar sizes exhibit superparamagnetic behaviour as evidenced by magnetic measurements of synthesized magnetoferritin [11]. 


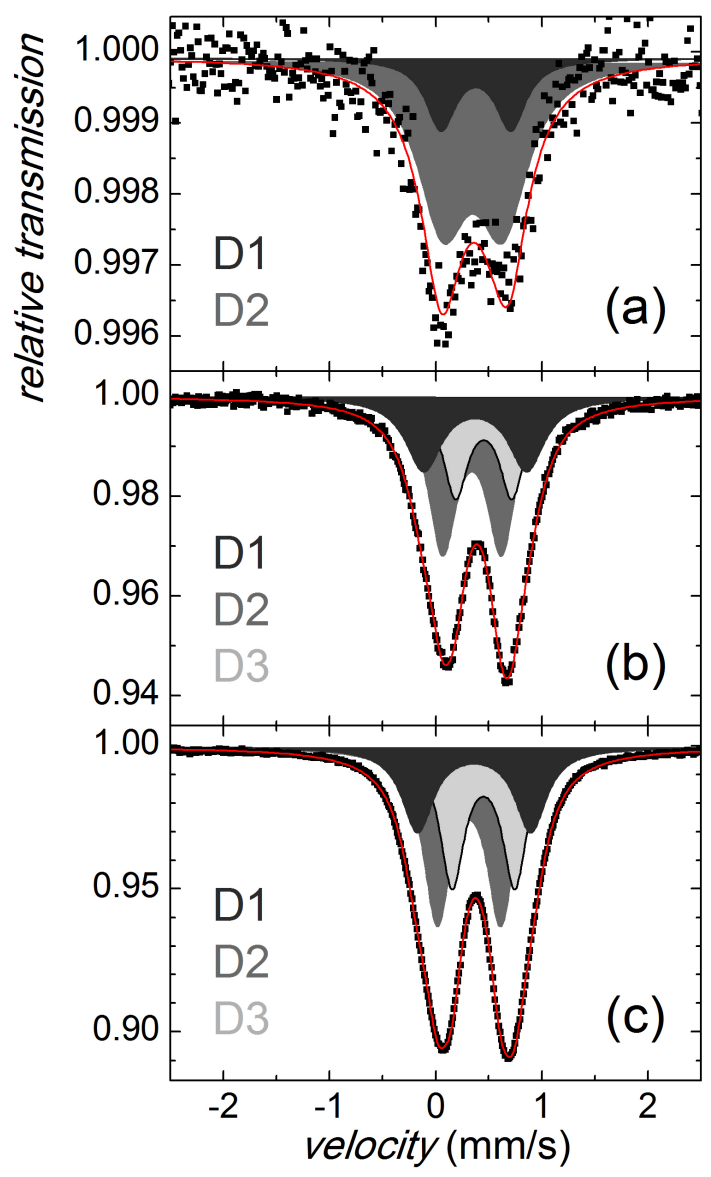

Fig. 1. Mössbauer spectra of human brain (a), human spleen (b), and horse spleen (c) measured at room temperature.

TABLE I

Parameters derived from the Mössbauer spectra of biological tissues that were recorded at room temperature including relative area $A[\%]$, isomer shift $I S[\mathrm{~mm} / \mathrm{s}]$, quadrupole splitting $Q S[\mathrm{~mm} / \mathrm{s}]$ and linewidth $\Gamma[\mathrm{mm} / \mathrm{s}]$.

\begin{tabular}{c|c|c|c|c}
\hline \hline Component & $A$ & $I S$ & $Q S$ & $\Gamma$ \\
\hline \multicolumn{5}{c}{ Human brain } \\
\hline D1 & 21 & 0.38 & 0.66 & 0.34 \\
D2 & 79 & 0.35 & 0.57 & 0.57 \\
\hline \multicolumn{5}{c}{ Human spleen } \\
\hline D1 & 28 & 0.37 & 0.97 & 0.41 \\
D2 & 46 & 0.34 & 0.55 & 0.32 \\
D3 & 26 & 0.45 & 0.53 & 0.28 \\
\hline \multicolumn{5}{c}{ Horse spleen } \\
\hline D1 & 25 & 0.36 & 1.06 & 0.36 \\
D2 & 44 & 0.31 & 0.60 & 0.31 \\
D3 & 31 & 0.45 & 0.59 & 0.28
\end{tabular}

The Mössbauer parameters of room-temperature doublets are listed in Table I. They can be assigned to hematite (D1) and ferrihydrite (D2) [6]. Doublet D3 can be due to magnetite and/or maghemite [10]. In the brain sample, D2 probably represents both hematite and ferrihydrite. No traces of sextets were revealed. It is noteworthy that X-ray diffractograms performed upon a sample of human spleen showed featureless broadened reflections [10] that are characteristic for a XRD amorphous material. They also confirm that the structure of ferritin particles is highly disordered and/or consists of particles with very small dimensions. Consequently, superparamagnetic behaviour of the latter can be expected.

As far as the relative amount of iron in the investigated tissues is concerned, we can estimate it from the scales of $y$-axes in Fig. 1 as well as from the scatter of experimental data. The lowest Fe content, which is at the limit of this technique, is seen in the sample of human brain. Sufficient quantity of Fe is found in the spleen samples. They also exhibit rather close hyperfine spectral parameters which suggest structural similarities between these two biological tissues. In all samples, structural positions of $\mathrm{Fe}$ in ferrihydrite are very alike as demonstrated by the respective hyperfine parameters of the D2 doublet.

Changes in the magnetic ordering of the investigated samples were observed after low-temperature measurements. The Mössbauer spectra contain along with sextets also one doublet. The resulting Mössbauer spectra of human brain, human and horse spleens are plotted in Fig. 2. Their decomposition into individual spectral components is also shown. Doublet-like components in the spectra obtained at $4.2 \mathrm{~K}$ indicate presence of very small particles with blocking temperature lower than $4.2 \mathrm{~K}$. They also demonstrate differences among the investigated samples from the point of view of superparamagnetic behaviour of this component. While its presence in the horse spleen sample is negligible it constitutes a significant portion in the human one and sufficiently well recognized doublet in the brain sample.

TABLE II

Parameters derived from the Mössbauer spectra of biological tissues that were recorded at $4.2 \mathrm{~K}$ including relative area $A[\%]$, isomer shift $I S[\mathrm{~mm} / \mathrm{s}]$, quadrupole splitting/shift $Q S[\mathrm{~mm} / \mathrm{s}]$, hyperfine magnetic field of the sextet components $B_{h f}[\mathrm{~T}]$, and linewidth $\Gamma[\mathrm{mm} / \mathrm{s}]$.

\begin{tabular}{c|c|c|c|c|c}
\hline \hline Component & $A$ & $I S$ & $Q S$ & $B_{h f}$ & $\Gamma$ \\
\hline \multicolumn{6}{c}{ Human brain } \\
\hline S1 & 59 & 0.45 & -0.06 & 50.23 & 0.59 \\
S2 & 12 & 0.46 & -0.13 & 48.16 & 0.42 \\
S3 & 17 & 0.45 & -0.14 & 44.05 & 0.73 \\
D1 & 12 & 0.44 & 0.60 & - & 0.34 \\
\hline \multicolumn{6}{c}{ Human spleen } \\
\hline S1 & 23 & 0.47 & -0.12 & 50.88 & 0.51 \\
S2 & 18 & 0.48 & -0.12 & 49.13 & 0.46 \\
S3 & 17 & 0.45 & -0.11 & 46.57 & 0.75 \\
D1 & 42 & 0.41 & 0.47 & - & 0.56 \\
\hline \multicolumn{6}{|c}{ Horse spleen } \\
S1 & 13 & 0.51 & -0.04 & 51.57 & 0.39 \\
S2 & 45 & 0.49 & -0.07 & 49.74 & 0.53 \\
S3 & 26 & 0.47 & -0.09 & 47.57 & 0.55 \\
S4 & 14 & 0.42 & -0.05 & 44.25 & 0.81 \\
D1 & 2 & 0.45 & 0.54 & - & 0.45
\end{tabular}




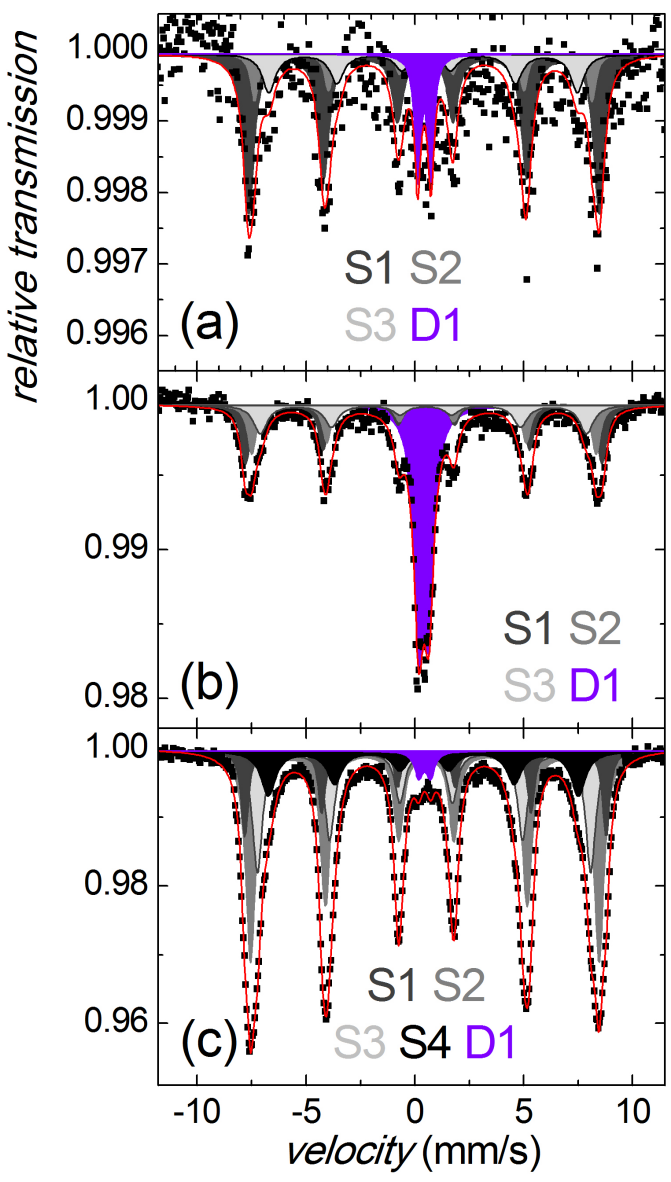

Fig. 2. Mössbauer spectra of human brain (a), human spleen (b), and horse spleen (c) measured at $4.2 \mathrm{~K}$.

The derived values of the spectral parameters including line area $(A)$, isomer shift $(I S)$, quadrupole shift/splitting $(Q S)$, hyperfine magnetic field $\left(B_{h f}\right)$, and line width $(\Gamma)$ are listed in Table II. The corresponding errors are of $\pm 1.5 \%, \pm 0.02 \mathrm{~mm} / \mathrm{s}, \pm 0.04 \mathrm{~mm} / \mathrm{s}, \pm 0.15 \mathrm{~T}$, and $\pm 0.04 \mathrm{~mm} / \mathrm{s}$, correspondingly.

We assign the individual spectral components as follows [10]: S1 - hematite, S2, S3 - ferrihydrite core and surface, respectively, S4 - magnetite and/or maghemite. The latter can be partially included also in S3 of the brain and human spleen samples. To distinguish between magnetite and maghemite only from the current Mössbauer spectrometry data is not so straightforward because the obtained hyperfine parameters deviate from those of pure substances presumably due to size and substitutional effects as evidenced by relatively large values of linewidths $(\Gamma \gtrsim 0.75 \mathrm{~mm} / \mathrm{s})$ as seen in $\mathrm{S} 3$ and $\mathrm{S} 4$ spectral components listed in Table II. Unfortunately, XRD turned out to be of a limited use for their identification, too [10]. D1 probably represents very small ferrihydrite and/or magnetite/maghemite particles.

\section{Conclusions}

Magnetic behaviour of Fe-containing nanoparticles that are found in biological tissues was studied by the help of ${ }^{57} \mathrm{Fe}$ Mössbauer spectrometry. While at room temperature the obtained Mössbauer spectra show exclusively doublet-like character, they are split into sextets after lowering the temperature of measurement to $4.2 \mathrm{~K}$. The spectra pointed out similarities among the investigated biological tissues as far as identification of individual structural components is concerned. Tough having slightly different hyperfine spectral parameters, basically the same structures, viz. hematite, ferrrihydrite, and magnetite and/or maghemite were revealed in all biological samples of different origin.

In order to characterize the particular structures more precisely, one has to consider Mössbauer experiments at low temperature with applied external magnetic field. Because of extremely low concentration of iron namely in human brain tissues such experiments are very demanding. In fact, even low temperature Mössbauer spectra of brain tissue are rather rare in a literature [2, 7]. On the other hand, namely the Mössbauer spectrometry is capable of providing evidence on local structural and/or magnetic arrangement of iron-containing nanoparticles in biological tissues.

\section{Acknowledgments}

This work was supported by the VEGA grant $1 / 0836 / 15$.

\section{References}

[1] R. Crichton, Inorganic Biochemistry of Iron Metabolism: From Molecular Mechanisms to Clinical Consequences, Wiley, England 2001.

[2] J. Galazka-Friedman, A. Friedman, E.R. Bauminger, Hyperfine Interact. 189, 31 (2009).

[3] U. Gonser, R.W. Grant, Biophys. J. 5, 823 (1965).

[4] M.I. Oshtrakh, I.V. Alenkina, A.V. Vinogradov, T.S. Konstantinova, V.A. Semionkin, Hyperfine Interact. 230, 123 (2015).

[5] I.V. Alenkina, M.I. Oshtrakh, Yu.V. Klepovac, S.M. Dubiel, N.V. Sadovnikov, V.A. Semionkin, Spectrochim. Acta A Mol. Biomol. Spectrosc. 100, 88 (2013).

[6] D. Meyrick, J. Webb, C. Cole, Inorg. Chim. Acta 339, 481 (2002).

[7] A. Friedman, J. Galazka-Friedman, E.R. Bauminger, D. Koziorowski, J. Neurol. Sci. 248, 31 (2006).

[8] M. Miglierini, R. Boča, M. Kopáni, A. Lančok, Acta Phys. Pol. A 126, 240 (2014).

[9] T. Zak, Y. Jiraskova, Surf. Interface Anal. 38, 710 (2006).

[10] M. Miglierini, J. Dekan, M. Kopani, A. Lancok, J. Kohout, M. Cieslar, AIP Conf. Proc. 1489, 107 (2012).

[11] Z. Mitróová, L. Melníková, J. Kováč, M. Timko, P. Kopčanský, Acta Phys. Pol. A 121, 1318 (2012). 